1 This manuscript is a preprint and has been submitted for publication at Fronteirs in Environmental Science. 2 Subsequent versions may have slightly different content. The DOI of the peer reviewed publication will be

3 provided if accepted. Please contact the authors if you have any questions or comments on this manuscript. 



\title{
Will it float? Rising and settling velocities of common macroplastic foils
}

\author{
Boaz Kuizenga ${ }^{1, *}$, Tim van Emmerik ${ }^{1}$, Kryss Waldschläger ${ }^{1}$, and Merel Kooi ${ }^{2}$ \\ ${ }^{1}$ Wageningen University and Research, Hydrology and Quantative Water \\ Management Group, Wageningen, The Netherlands \\ ${ }^{2}$ Wageningen University and Research, Aquatic Ecology and Water Quality Group, \\ Wageningen, The Netherlands \\ Correspondence*: \\ Boaz Kuizenga \\ boaz.kuizenga@wur.nl
}

\section{ABSTRACT}

Plastic accumulates in the environment because of insufficient waste handling and the materials' high durability. Better understanding of plastic behaviour in the aquatic environment is needed to estimate transport and accumulation, which can be used for monitoring strategies, prevention measures, and plastic clean-up activities. Plastic transport models benefit from accurate description of particle characteristics, such as rising and settling velocities. For macroplastics, these are however still scarce. In this research, the rising and settling behaviour of three different polymer types (PET, PP, and PE) was investigated, which are the most common in the environment. All of the plastic particles were foils of different surface areas. A new method for releasing rising plastics without interfering the flow and disturbing the column was used. Four models that estimate the velocity based on the characteristics of the plastics are discussed, of which three are from literature, and one is newly derived. These models are validated using the data generated in this research, and data from another study on rising and settling velocities of plastic. From the models that were discussed, the best results are from the newly introduced velocity model for foils ( $R^{2}=0.96$ and 0.58 , for both datasets). This model shows potential to estimate the rising and settling velocity of plastics, and should be examined further by using additional data. The results of our paper can be used to further explore the vertical distribution of plastics in rivers, lakes and oceans, which is crucial to optimize future monitoring and cleanup efforts.

Keywords: environmental fluid mechanics, experimental, marine debris, plastic pollution, microplastic, hydrology, hydrodynamics

\section{INTRODUCTION}

Plastics have a high durability, are light weight and cheap to manufacture, which makes them a popular resource for a variety of products. Because of the high durability, it does not decompose easily and stays in the environment for a long time. This results in accumulation of plastic waste in the environment (Barnes et al., 2009; Lebreton et al., 2018)

Rivers and oceans are polluted by plastic waste. Rivers transport the land-based plastic towards the sea, and plastic pollution causes environmental damage to the river's ecosystems (Emmerik and Schwarz, 2020; Meijer et al., 2021). To manage and prevent the waste streams of plastics in rivers, it is necessary 
to better understand their behavior in freshwater. More specifically, few is known about the vertical distribution of macroplastics below the surface. A theoretical approach to estimate the vertical distribution of plastics will complement the development of observation-based methods, for example new monitoring techniques, empirical methods, and other approaches for under water plastic estimates (Broere et al., 2021; Van Emmerik et al., 2019).

Rising and settling velocities give an indication of the vertical movement of plastics. The terminal velocity of particles is one of the main parameters when it comes to sedimentation models (Dietrich, 1982). Knowing the terminal rising and settling velocities allows a better selection of plastic cleanup strategies (Helinski et al., 2021), which may depend on the vertical distribution of plastics. The vertical velocities depend on the properties of the plastics, and will determine the fate of the particles. Therefore, a better understanding is needed to understand how particles move in water, and where for example sedimentation hot-spots will occur.

Most research that is done on the rising and settling velocities focused on microplastics (plastics with a diameter $\leq 5 \mathrm{~mm}$ ) in salt water (Kaiser et al., 2017; Kowalski et al., 2016; Reisser et al., 2015; Kooi et al. 2016; Ballent et al., 2012). There has been some research done on rising and settling velocities of microplastics in fresh water (Waldschläger and Schüttrumpf, 2019; Khatmullina and Isachenko, 2017), but there is no systematic research done for a range of macropastics. The research that is done on macroplastics (plastics with a diameter $>5 \mathrm{~mm}$ ) in fresh water (Waldschläger et al., 2020) focussed on plastic collected from the environment, and did not consider different shapes and surface areas of the same polymers. Therefore, a systematic analysis of rising and settling velocities of macroplastic in fresh water is needed to gain a the better understanding of the plastic transport in natural systems.

Here, we systematically performed rising and settling velocity measurements on foils (a minimum thickness:length:width ratio of 1:16:16 (Kooi and Koelmans, 2019)), for three different polymers. Foils were selected as this shape is only rarely addressed in current research (Van Melkebeke et al., 2020). Furthermore, four different models that calculate the theoretical velocity based on the parameters of the plastics are reviewed based on this dataset and the dataset of Waldschläger et al. (2020). Three of these models are from literature (Ferguson and Church, 2004; Le Roux, 2002, Stokes, 1851), and one was newly developed.

Every model is different, but they all base on the same characteristics of the particles and fluid: fluid density, and particle properties such as density, shape and diameter. Foils behave differently than other, more spherical particles, and it is therefore the question if these models are suitable to estimate rising and settling velocities for macroplastic foils (Van Melkebeke et al., 2020). With this paper we present (1) a laboratory method to perform macroplastic settling/velocity measurements, and (2) a new model to theoretically determine the velocity based on the item characteristics.

\section{MATERIALS AND METHODS}

Three different polymer types are systematically researched on their rising or settling velocity. Furthermore, four different models are reviewed on their ability to estimate the rising and settling velocity of the plastics.

\subsection{Plastic item selection}

In this study, we focused on the three most abundant plastic types found in rivers, namely polyethylene terephthalate (PET), polypropylene (PP), and polyethylene (PE) (Schwarz et al., 2019). PET has a density higher than water $\left(1370 \mathrm{~kg} / \mathrm{m}^{3}<\rho<1450 \mathrm{~kg} / \mathrm{m}^{3}\right.$ (Hidalgo-Ruz et al., 2012)) and will therefore sink in natural, stagnant waters. PE and PP have densities lower than water $\left(910 \mathrm{~kg} / \mathrm{m}^{3}<\rho<970 \mathrm{~kg} / \mathrm{m}^{3}\right.$ and 900 

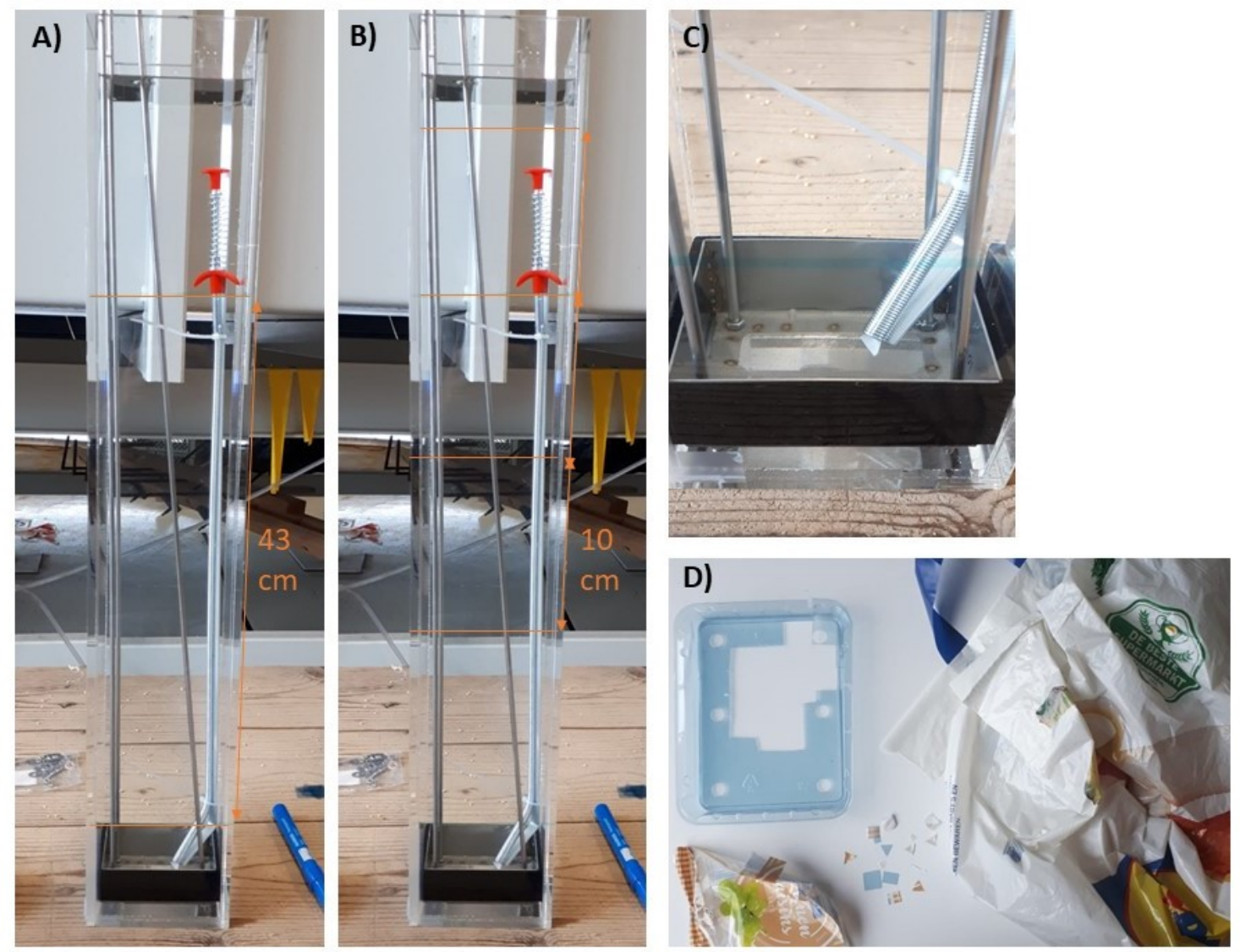

Figure 1. (A) The set-up for the settling velocity measurements. The lines indicate the start and stop line for the stopwatch. The basket for retrieving the particles is visible at the bottom. (B) The set-up for the rising velocity measurements. The lines indicate the start and stop lines for the stopwatch. (C) Close-up of the claw mechanism, which is holding a piece of plastic in place during the measurements. (D) All sampled items for the experiments: the mushroom cover (PET) on the top left, the plastic bag (PE) on the right and the raisin packaging (PP) on the bottom left.

$\mathrm{kg} / \mathrm{m}^{3}<\rho<910 \mathrm{~kg} / \mathrm{m}^{3}$, respectively (Hidalgo-Ruz et al., 2012)) and will therefore rise when submerged in the water column. The plastics were bought in the supermarket. For PET, the lid of a mushroom box was used; for PP a raisin packaging and for PE a shopping bag. These were manually cut in different shapes and sizes (table 1, figure 1D).

\subsection{Experiment set-up}

The measurements were done in an acrylate column with an inside footprint of $10 x 10 \mathrm{~cm}$ and a height of $70 \mathrm{~cm}$ (figure 1 A), filled with tap water. The particle sizes were chosen, such that there would be no influence of the wall of the column on the measurements (the wall was not touched by the particle during the run). The average settling and rising time of the plastics was recorded over a certain vertical length. A previous study, using similarly sized plastics, showed that plastics reach their terminal velocity within 15 $\mathrm{cm}$ (Waldschläger et al., 2020). To be sure, the first $20 \mathrm{~cm}$ of the column was used for acceleration of the plastic in this research. This was done for both rising and settling velocity measurements.

\subsubsection{Settling velocity}

The particles were released in the water column completely submerged, to make sure that no air bubbles were attached to the plastics and that they would not float because of the surface tension of the water. For the settling velocity measurements, a basket was put at the bottom to make it easier to pick up the particles 
Table 1. Overview of measurements that were done. $\mathrm{R}=$ rectangle, $\mathrm{T}=$ triangle.

\begin{tabular}{lllll} 
Material & Surface area $\left[\mathrm{cm}^{2}\right]$ & Shape & L x W $[\mathrm{cm}]$ & \# measurements \\
\hline PET & 1.25 & $\mathrm{R}$ & $1 \times 1.25$ & 10 \\
& 1 & $\mathrm{R}$ & $1 \times 1$ & 10 \\
& 0.5 & $\mathrm{~T}$ & $1 \times 1$ & 12 \\
& 0.25 & $\mathrm{R}$ & $0.5 \times 0.5$ & 10 \\
\hline $\mathrm{PP}$ & 1.25 & $\mathrm{R}$ & $1 \times 1.25$ & 11 \\
& 1 & $\mathrm{R}$ & $1 \times 1$ & 10 \\
& 0.5 & $\mathrm{~T}$ & $1 \times 1$ & 10 \\
& 0.25 & $\mathrm{R}$ & $0.5 \times 0.5$ & 11 \\
$\mathrm{PE}$ & 0.075 & $\mathrm{R}$ & $0.05 \times 1.5$ & 10 \\
& 1.25 & $\mathrm{R}$ & $1 \times 1.25$ & 10 \\
& 1 & $\mathrm{R}$ & $1 \times 1$ & 10 \\
& 0.5 & $\mathrm{~T}$ & $1 \times 1$ & 10 \\
& 0.25 & $\mathrm{R}$ & $0.5 \times 0.5$ & 16 \\
& 0.075 & $\mathrm{R}$ & $0.05 \times 1.5$ & 10
\end{tabular}

89 after the measurements, and the same item could be measured repeatedly (figure 1 A). After the particles were retrieved from the water column, the basket was put back into the column. To make sure the water column was stagnant, the new measurements were only done if the water column appeared stable, but at least after 1 minute. A stopwatch was started when the particle reached the line $20 \mathrm{~cm}$ below the water surface. The bottom line - where the stopwatch was stopped - was placed at the lowest possible position, without having the basket interfere with the particles. This resulted in a distance of $43 \mathrm{~cm}$ over which the measurement was conducted (figure 1 A).

\subsubsection{Rising velocity}

For the rising velocity measurements, the water column was divided in six areas (from the bottom up): an acceleration part of $20 \mathrm{~cm}$, four measurement parts of each $10 \mathrm{~cm}$, and the excess part. These four measurements per particle were only done for the rising velocity measurements (figure 1B).

Because the measurements are done in a stable water column, a release mechanism at the bottom of the column is required for rise velocity measurements. Previous methods for releasing the plastics were too difficult for macroplastics, or did not inquire a stagnant water column (Waldschläger and Schüttrumpf, 2019; Zaat, 2020). That is why, for the rising velocity, a new method for releasing the particle was made. The new method consists of a flexible 'claw' mounted onto an aluminium frame (figure $1 \mathrm{C}$ ). The claw is held into a corner, making it possible to release the plastics without interfering the flow. By pushing on top of the claw, the hook releases the plastic without having to disturb the water. This way, the water remains as stagnant as possible.

First, a test run was done for the plastic, to determine the position of the release mechanism and the time it takes for the plastic to reach the surface. Depending on this time, the distance over which the plastic was measured, was chosen. The four $10 \mathrm{~cm}$ lines (figure 1B) were taken together in either parts of 20 or $40 \mathrm{~cm}$ if the plastic was fast to make sure the measurements are precise. Measurements of $10 \mathrm{~cm}$ were chosen if the plastic was slow. So, if $10 \mathrm{~cm}$ was chosen, then for one run the time was recorded four times.

\subsection{Model evaluation}

To estimate the rising and settling velocities of other plastics, mathematical models are used. These models all base their velocity on the size, shape, and density of the particle. Also, the properties of the water such as viscosity and density are taken into account. The dynamic viscosity was estimated using the measured temperature of the water. For all theoretical velocities, the density of water was estimated at 
$999 \mathrm{~kg} / \mathrm{m}^{3}$. The density of the plastics were obtained from Hidalgo-Ruz et al. (2012)(section 2.1). From the range mentioned in the article, the mean was taken as a density for each polymer type.

To get a better view on the validity of the models, two datasets are used. One is the dataset derived in this research, and the other is the data from Waldschläger et al. (2020), which includes mainly microplastics of different shapes.

The Reynolds number can give an indication for the turbulence of the flow. Depending on the turbulence of the flow, assumptions in the models can be made. Because some models make assumptions that are based on the turbulence of the flow, the Reynolds numbers for all polymers were calculated, using equation 1. This can give an indication of the applicability of the models.

$$
R e=\frac{v \cdot d \cdot \rho}{\mu}
$$

In equation 1, $d$ is the equivalent diameter of the particle in $m, \rho$ the density of water in $\mathrm{kg} / \mathrm{m}^{3}, \mu$ the dynamic viscosity of water in $\mathrm{Pa} / \mathrm{s}$ and $v$ the velocity of the particle in $\mathrm{m} / \mathrm{s}$.

A theoretical settling velocity was calculated for all plastic items, given the parameters above and the plastic size and density. When these theoretical velocities and the measured data are plotted against each other, the points should lie on the line $y=x$. To be able to visually interpret the quality of the model, the $y=x$ line is plotted (black line) as a reference in every plot. To calculate how the model represented the $y=x$ line, an $R^{2}$ was calculated. The closer this value is to 1 , the closer the model is to the $y=x$ line, thus the better the model is.

The four models that are reviewed are: 1) the Stokes model for laminar flow (Stokes, 1851), 2) a model based on both laminar and turbulent flow (Ferguson and Church, 2004), 3) a settling velocity model based on the Hofmann shape entropy (Hofmann, 1994; Le Roux, 1997, 2002), and 4) a model based on the turbulent drag force, derived in this research.

These models base their velocity on a shape factor, or on a constant that is empirically determined, in which the shape of the particle plays a role. This is relevant, because the particles measured in this research have a shape that is not found in natural grains often. Therefore, the value of these models for platy particles and foils is researched.

The first model for settling velocity that was reviewed, was the Stokes equation for settling velocity (equation 2). Stokes derived this from the simplified Navier-Stokes equations. Although this relation can only be used for very low Reynolds numbers (Waldschläger et al., 2020), the Stokes equation forms the basis for a lot of models for settling velocity of natural grains, and is thoroughly researched. It can also be used for plastic, at least in an adjusted form (Ferguson and Church, 2004; Gibbs et al., 1971).

$$
v=\frac{2}{9} \cdot r^{2} \cdot g \cdot \frac{\left(\rho_{p}-\rho_{f}\right)}{\mu}
$$

In this equation, $r$ is the equivalent sphere radius (ESR) of the particle in $m, g$ is the gravitational acceleration in $\mathrm{m} / \mathrm{s}^{2}, \mu$ the dynamic viscosity of water in $\mathrm{Pa} / \mathrm{s}$, and $\rho_{p}$ and $\rho_{f}$ are the density of the particle and the fluid in $\mathrm{kg} / \mathrm{m}^{3}$, respectively. The equivalent sphere radius was calculated using the volume of the particles, and relating that volume to a sphere. The more the particle shape deviates from a sphere, the worse this equivalent radius estimation gets. That is why the Stokes equation works best for perfect spheres. 
A different equation for settling velocity was developed by Ferguson and Church (2004):

$$
v=\frac{R \cdot g \cdot D^{2}}{C_{1} \cdot \nu+\left(0.75 \cdot C_{2} \cdot R \cdot g \cdot D^{3}\right)^{0.5}}
$$

155 In which $R=\frac{\rho_{p}-\rho_{f}}{\rho_{f}}$ (submerged specific gravity), $D$ is the equivalent diameter of the particle in $\mathrm{cm}$, and $g$ 156 is the gravitational acceleration in $\mathrm{m} / \mathrm{s}^{2}$. For the polymers with a density lower than water, the submerged 157 specific gravity was taken absolute in the denominator, because of the power 0.5 . The constants $C_{1}$ (constant 158 from Stokes' law for laminar settling) and $C_{2}$ (drag coefficient for Reynolds numbers exceeding $10^{3}$ ) are 159 based on the shape of the particle and the properties of the fluid. The difference with the Stokes model 160 is that this model incorporates a factor for turbulent flow, and is therefore applicable at a larger range of 161 Reynolds numbers.

162 For smooth spheres, $C_{1}$ and $C_{2}$ were determined to be 18 and 0.4 respectively, but for particles with other 163 shapes these values will become higher. In this research, values of 24 for $C_{1}$ and 1.2 for $C_{2}$ were assumed, 164 as these are the theoretical limit for very angular grains for this model (Ferguson and Church, 2004). Same 165 as in the Stokes equation 2, for this equation the diameter was calculated using the ESR. This equation 166 combines Stokes' law for laminar flow with the turbulent drag, and can therefore be used for Reynolds 167 numbers up to 100,000 (Ferguson and Church, 2004).

168 A third theoretical approach is based on the Hofmann Shape Entropy (HSE), which was formulated by 169 Hofmann (1994). The HSE is a shape factor which describes the shape of a particle, with 1 being a perfect 170 sphere.

According to Van Melkebeke et al. (2020), no shape factor can differentiate between foils, fibres and granular particles, but they can be used to describe particles within a certain shape. The velocity model based on the HSE is mainly used for ellipsoid particles (Le Roux, 1997), but can also be used for irregular shaped grains (Le Roux, 2002). In this research, equation 4 was used, which was derived by Le Roux (2002):

$$
v=v_{\text {sphere }} \cdot \frac{H S E-0.23}{0.77}
$$

In equation 4, $v_{\text {sphere }}$ is the theoretical velocity (in $\mathrm{m} / \mathrm{s}$ ) if the particle is a perfect sphere (which was derived in Le Roux (1992)), and the constants are empirical. Because of the HSE and the constants, this model can be used for other shapes as well. This set of equations can be used for $R e<100,000$ (Le Roux, 1997, 2002). Equation 4 is the end product of this derivation.

The last equation that was used in this research, is named the velocity model for foils (Equation 10$)$. This equation is derived for this research.

The velocity model follows from the idea that when the gravity force (eq. 5), buoyancy force (eq. 6), and the drag force (eq.7) are equal, the particle reaches its terminal velocity.

$$
\begin{aligned}
& F_{g}=L \cdot B \cdot D \cdot g \cdot \rho_{p} \\
& F_{b}=L \cdot B \cdot D \cdot g \cdot \rho_{f}
\end{aligned}
$$


184

\section{RESULTS AND DISCUSSION} negative velocities because of the square root. results from this research are more reliable. not inquired.

$$
\begin{gathered}
F_{D}=\frac{1}{2} \cdot \rho_{f} \cdot v^{2} \cdot C_{D} \cdot A \\
\frac{1}{2} \cdot \rho_{f} \cdot v^{2} \cdot C_{D} \cdot A=L \cdot B \cdot D \cdot g \cdot\left(\rho_{p}-\rho_{f}\right) \\
v=\sqrt{2 \cdot D \cdot g \cdot \frac{\rho_{p}-\rho_{f}}{\rho_{f} \cdot C_{D}}}
\end{gathered}
$$

It was observed that during the settling velocity experiment, the foils came down with a swaying, sideways motion. Because of this, it is assumed that the thickness D can better be approximated with the ESR ('r' in the equation) times the CSF, which is the shape factor defined by Corey (1949) and McNown and Malaika (1950). This results in the final velocity model for foils:

$$
v=I_{B}+C_{B} \cdot \sqrt{2 \cdot r \cdot C S F \cdot g \cdot \frac{\rho_{p}-\rho_{f}}{\rho_{f} \cdot C_{D}}}
$$

In equation 10, $r$ is the equivalent radius in $m, g$ is the gravitational acceleration in $m / s^{2}, \rho_{f}$ and $\rho_{p}$ are the density of the fluid and the particle in $\mathrm{kg} / \mathrm{m}^{3}$, and $C_{B}$ and $I_{B}$ are empirical constants. The radius of the particles was calculated in the same way as for the other equations. The drag constant $C_{D}$ was assumed at 1.5, because the particles are platy and will thus have a lot of turbulent drag (Hoerner, 1965). For this equation, the measured velocity was transformed to an absolute velocity, since equation 10 can not model

As this model was derived from theory, two empirical constants were introduced $\left(C_{B}\right.$ and $\left.I_{B}\right)$ to make the best fit for this model. This was done by performing a linear regression analysis. Firstly, the constant $C_{B}$ was assumed at 1 , and $I_{B}$ was assumed to be 0 (that is true if the model is perfect). After this, the model was corrected for the slope of the model with the old constants, using the regression result. By assigning new values for the constants, the model was changed to obtain a better fit with the measured data. The model was validated using the data from Waldschläger et al. (2020). In this study, for 100 particles collected from a fluvial environment, the rising or settling velocity is measured. This dataset ranges from microplastic to small macroplastic particles of different polymer types.

\section{The observed settling velocities for PET are in the range of 0.029 to $0.037 \mathrm{~m} / \mathrm{s}$, for PE the observed rising} velocities are in the range of 0.0001 to $0.004 \mathrm{~m} / \mathrm{s}$, and for PP the observed rising velocities are in the range of 0.002 and $0.006 \mathrm{~m} / \mathrm{s}$. In table 2, the results and assumptions of all the models are summarized. In contrast to other research on rising velocity of plastics (Zaat, 2020; Kooi et al., 2016), this research included a new method for the plastic release without disturbing the water column. This means that the

A lot of research on environmental plastics are done on microplastics (Kooi et al., 2016, Reisser et al. 2015; Khatmullina and Isachenko, 2017; Waldschläger and Schüttrumpf, 2019), but to date not much research has been done on macroplastics (Waldschläger et al., 2020, Zaat, 2020). Zaat (2020) performed measurements on large pieces of low and high density PE, but in these experiments, a stable column was 
The plastics were - in contrary to nature - not in water for at least a few hours before the velocity was measured. This has a large impact on the rising and settling velocity of microplastics (Kaiser et al., 2017), however the impact on macroplastics is not yet determined. Furthermore, in the environment biofouling and particle aggregation will take place, which will change the behaviour of the plastics even further (Van Melkebeke et al., 2020, Michels et al., 2018).

The Reynolds number is a measure for turbulence (Equation 1). The Reynolds regime of this experiment falls in the following range: $12<R e<10,000$. The four models that were used in this study are valid for different Reynolds regimes (table 2)(Stokes, 1851; Ferguson and Church, 2004; Le Roux, 2002). Stokes equation gives only an inaccurate approximation, because that model is most suited for very low Reynolds numbers because of the assumptions made in the derivation (Stokes, 1851). The other models do work for this regime, and are therefore more suitable to be applied to the data.

\begin{tabular}{c|c|c|c|c} 
Model & Re regime & Shape factor & $\mathrm{R}^{2}$ with $\mathrm{y}=\mathrm{x}(1)$ & $\mathrm{R}^{2}$ with $\mathrm{y}=\mathrm{x}(2)$ \\
\hline Stokes & $<1$ & - & -0.17 & -0.11 \\
\hline Ferguson and Church & $<100.000$ & Integrated in constants & 0.58 & -0.73 \\
\hline Le Roux & $<100.000$ & HSE & -0.99 & $-2 \mathrm{E5} 1$ \\
\hline VMF with constants & Turbulent regime & CSF & 0.96 & 0.58 \\
\hline VMF, no constants & Turbulent regime & CSF & -0.37 & -0.79
\end{tabular}

Table 2. Summary of the researched velocity models and their assumptions. VMF $=$ velocity model for foils. (1) is the dataset from this research, (2) is the dataset from Waldschläger et al. (2020)

All models discussed are plotted against the measured velocities from the datasets. The plots for the models from literature are available in the supplementary information, the plots for the new model are shown in figure 2. The model that is based on turbulent drag is presented in figure 2. This model was calibrated with the data generated in this research, and therefore responds best from all models on this dataset. Two empirical constants were introduced to fit the data better, which have the values of $C_{B}=1.96$ and $I_{B}=-0.004$. Because equation 10 has a square root, the results of the rising velocity experiments were taken absolute. This could give a different value for the constants $C_{B}$ and $I_{B}$.

In Waldschläger and Schüttrumpf (2019), six models from sedimentation theory are researched for microplastics. The Stokes model was also researched in that model, but the others are different. In Waldschläger and Schüttrumpf (2019), the models are found to estimate the behaviour of all particles with insufficient precision. The same was found for the models from literature in this research, based on the data for macroplastics. The new model from this research shows promising results, and should be researched further.

Van Melkebeke et al. (2020) researched different shape factors on their ability to describe different plastic shapes. They found that no shape factor is able to describe all different kinds of particles, and therefore no model in this research would be able to describe all sorts of plastic. However, a model can describe one type of plastic separately.

Our systematic laboratory research on macroplastic may be used as a basis for further research on macroplastics in the environment. The use of models is a valuable aspect of this research, and - if researched further - can contribute to a better understanding of the behaviour of plastics in the aquatic environment. Future research can be based on this study, but should be elaborated: for example more measurements with different plastics, flowing water, and different flow regimes can improve the capacity of the models. 

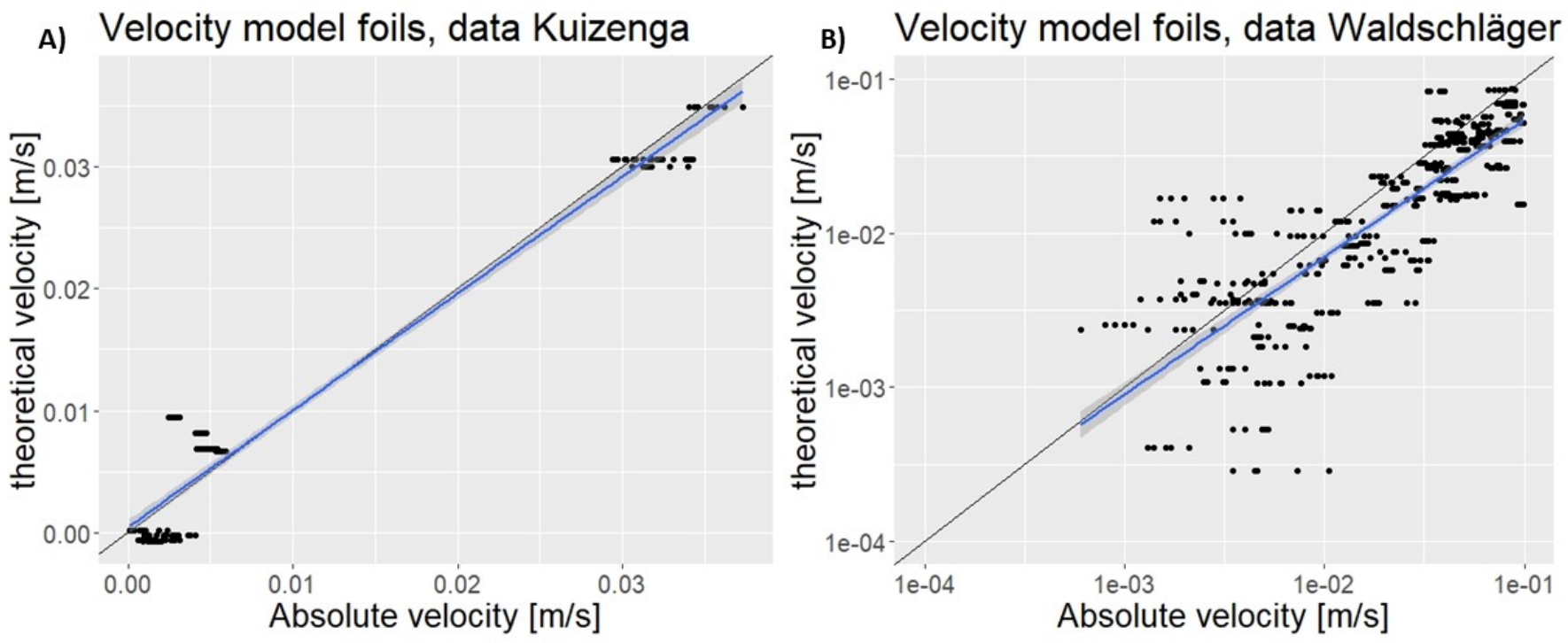

Figure 2. Velocity model for foils plotted with the data generated in this research (A) and the data from Waldschläger et al. (2020) (B). The line $y=x$ is shown as the black line.

\section{CONCLUSION}

In this research, three different polymer types and five different surface area classes were tested on their rising and settling behaviour. Three different models from literature and one model derived from theory were used to calculate the velocity. The newly developed technique to release the polymers with a density lower than water (i.e. the rising plastics) worked. This method, consisting of a claw and an aluminium frame, is easy to use and establish.

PET was found to have a relatively large settling velocity $(0.029-0.037 \mathrm{~m} / \mathrm{s})$. This could indicate that PET sinks to the bottom of a fresh-water system quite fast. However, the larger the PET foil is, the slower it will sink. PE and PP are found to rise relatively slow $(0.0001-0.004 \mathrm{~m} / \mathrm{s}$ and $0.002-0.006 \mathrm{~m} / \mathrm{s}$, respectively). This might indicate that they are part of the water column, and that they are more influenced by turbulent movements in the river.

From all four models that were introduced, only two estimated the behaviour of the platy particles relatively well based on the measured data: the model by Ferguson and Church $(2004)\left(R^{2}=0.58\right)$ and the model based on the drag force that was introduced in this research $\left(\mathrm{R}^{2}=0.96\right)$. As the empirical constants $C_{B}$ and $I_{B}$ differ with each polymer type, the exact value of these parameters should be further researched for every polymer. However, the values of 1.964 for $C_{B}$ and -0.0041 for $I_{B}$ that was found in this research, could give a good indication. All performed less when the data from Waldschläger et al. (2020) was used, compared to the data generated in this research. This is probably due to the bigger differences in shapes and sizes in the data from Waldschläger et al. (2020), which are harder to estimate using models. Despite this, the data generated and model analysis performed in this study are valuable for further plastic research. 


\section{FUNDING}

274

\section{SUPPLEMENTAL DATA}

\section{DATA AVAILABILITY STATEMENT}

\section{REFERENCES}

\section{CONFLICT OF INTEREST STATEMENT}

\section{ACKNOWLEDGMENTS} found in the Frontiers LaTeX folder. bgd-9-18755-2012 doi:10.1029/WR018i006p01615

With this paper we aim to shed new light on rising and settling velocities of common macroplastic items. We provide an experimental setup that can be used for future research, and developed a simple model to

Supplementary Material should be uploaded separately on submission, if there are Supplementary Figures, please include the caption in the same file as the figure. LaTeX Supplementary Material templates can be

The datasets generated for this study can be found in the 4TU research data repository: here,

Ballent, A., Purser, A., Mendes, P. D. J., Pando, S., and Thomsen, L. (2012). Physical transport properties of marine microplastic pollution. Biogeosciences Discussions 9, 18755-18798. doi:10.5194/

Barnes, D. K., Galgani, F., Thompson, R. C., and Barlaz, M. (2009). Accumulation and fragmentation of plastic debris in global environments. Philosophical Transactions of the Royal Society B: Biological Sciences 364, 1985-1998. doi:10.1098/rstb.2008.0205

Broere, S., van Emmerik, T., González-Fernández, D., Luxemburg, W., de Schipper, M., Cózar, A., et al. (2021). Towards underwater plastic monitoring using echo sounding , 1-18

Corey, A. (1949). Influence of shape on the fall velocity of sand grains. Ph.D. thesis

Dietrich, W. E. (1982). Settling velocity of natural particles. Water Resources Research 18, 1615-1626.

Emmerik, T. and Schwarz, A. (2020). Plastic debris in rivers. WIREs Water 7. doi:10.1002/wat2.1398

Ferguson, R. I. and Church, M. (2004). A simple universal equation for grain settling velocity. Journal of Sedimentary Research 74, 933-937. doi:10.1306/051204740933 
Gibbs, R. J., Matthews, M. D., and Link, D. A. (1971). The Relationship Between Sphere Size And Settling Velocity. SEPM Journal of Sedimentary Research Vol. 41, 1689-1699. doi:10.1306/ 74D721D0-2B21-11D7-8648000102C1865D

Helinski, O. K., Poor, C. J., and Wolfand, J. M. (2021). Ridding our rivers of plastic: A framework for plastic pollution capture device selection. Marine Pollution Bulletin 165, 112095. doi:10.1016/j. marpolbul.2021.112095

Hidalgo-Ruz, V., Gutow, L., Thompson, R. C., and Thiel, M. (2012). Microplastics in the marine environment: A review of the methods used for identification and quantification. Environmental Science and Technology 46, 3060-3075. doi:10.1021/es2031505

Hoerner, S. F. (1965). Fluid-dynamic drag (Bakersfield, CA). doi:http://resolver.tudelft.nl/uuid: c59c54da-4641-4344-b580-07ac2a31cc35

Hofmann, H. J. (1994). Grain-shape indices and isometric graphs. Journal of Sedimentary Research A: Sedimentary Petrology \& Processes, 916-920doi:10.1306/d4267f0a-2b26-11d7-8648000102c1865d

Kaiser, D., Kowalski, N., and Waniek, J. J. (2017). Effects of biofouling on the sinking behavior of microplastics. Environmental Research Letters 12. doi:10.1088/1748-9326/aa8e8b

Khatmullina, L. and Isachenko, I. (2017). Settling velocity of microplastic particles of regular shapes. Marine Pollution Bulletin 114, 871-880. doi:10.1016/j.marpolbul.2016.11.024

Kooi, M. and Koelmans, A. A. (2019). Simplifying Microplastic via Continuous Probability Distributions for Size, Shape, and Density. Environmental Science and Technology Letters 6, 551-557. doi:10.1021/ acs.estlett.9b00379

Kooi, M., Reisser, J., Slat, B., Ferrari, F. F., Schmid, M. S., Cunsolo, S., et al. (2016). The effect of particle properties on the depth profile of buoyant plastics in the ocean. Scientific Reports 6, 1-10. doi:10.1038/srep33882

Kowalski, N., Reichardt, A. M., and Waniek, J. J. (2016). Sinking rates of microplastics and potential implications of their alteration by physical, biological, and chemical factors. Marine Pollution Bulletin 109, 310-319. doi:10.1016/j.marpolbul.2016.05.064

Le Roux, J. P. (1992). Settling velocity of spheres: a new approach. Sedimentary Geology 81, 11-16. doi:10.1016/0037-0738(92)90053-T

Le Roux, J. P. (1997). Comparison of Sphericity Indices as Related to the Hydraulic Equivalence of Settling Grains. SEPM Journal of Sedimentary Research Vol. 67, 634. doi:10.1306/ D42685BD-2B26-11D7-8648000102C1865D

Le Roux, J. P. (2002). Application of the Hofmann shape entropy to determine the settling velocity of irregular, semi-ellipsoidal grains. Sedimentary Geology 149, 237-243. doi:10.1016/S0037-0738(01) 00175-0

Lebreton, L., Slat, B., Ferrari, F., Sainte-Rose, B., Aitken, J., Marthouse, R., et al. (2018). Evidence that the Great Pacific Garbage Patch is rapidly accumulating plastic. Scientific Reports 8, 1-15. doi:10.1038/s41598-018-22939-w

McNown, J. and Malaika, J. (1950). Effects of particle shape on settling velocity at low Reynolds numbers. American Geophysical Union 31, 74-82

Meijer, L. J., van Emmerik, T., van der Ent, R., Schmidt, C., and Lebreton, L. (2021). More than 1000 rivers account for $80 \%$ of global riverine plastic emissions into the ocean. Science Advances 7, 1-14. doi:10.1126/sciadv.aaz5803

Michels, J., Stippkugel, A., Lenz, M., Wirtz, K., and Engel, A. (2018). Rapid aggregation of biofilmcovered microplastics with marine biogenic particles. Proceedings of the Royal Society B: Biological Sciences 285. doi:10.1098/rspb.2018.1203 
Reisser, J., Slat, B., Noble, K., Du Plessis, K., Epp, M., Proietti, M., et al. (2015). The vertical distribution of buoyant plastics at sea: An observational study in the North Atlantic Gyre. Biogeosciences 12, 1249-1256. doi:10.5194/bg-12-1249-2015

Schwarz, A. E., Ligthart, T. N., Boukris, E., and van Harmelen, T. (2019). Sources, transport, and accumulation of different types of plastic litter in aquatic environments: A review study. Marine Pollution Bulletin 143, 92-100. doi:10.1016/j.marpolbul.2019.04.029

Stokes, G. G. (1851). On the Effect of the Internal Friction of Fluids on the Motion of Pendulums. The Transactions of the Cambridge Philosophical Society 9, 1-10. doi:10.1017/cbo9780511702266.002

Van Emmerik, T., Loozen, M., Van Oeveren, K., Buschman, F., and Prinsen, G. (2019). Riverine plastic emission from Jakarta into the ocean. Environmental Research Letters 14. doi:10.1088/1748-9326/ ab30e8

Van Melkebeke, M., Janssen, C., and De Meester, S. (2020). Characteristics and Sinking Behavior of Typical Microplastics including the Potential Effect of Biofouling: Implications for Remediation. Environmental Science and Technology 54, 8668-8680. doi:10.1021/acs.est.9b07378

Waldschläger, K., Born, M., Cowger, W., Gray, A., and Schüttrumpf, H. (2020). Settling and rising velocities of environmentally weathered micro- and macroplastic particles. Environmental Research 191. doi:10.1016/j.envres.2020.110192

Waldschläger, K. and Schüttrumpf, H. (2019). Effects of Particle Properties on the Settling and Rise Velocities of Microplastics in Freshwater under Laboratory Conditions. Environmental Science and Technology 53, 1958-1966. doi:10.1021/acs.est.8b06794

Zaat, L. A. (2020). Below the Surface (MSc thesis) 